- The nature and quality of periodontal-related patient information found on the internet is variable in content and quality.

- Commonly used internet search engines do not readily distinguish the 'wheat from the chaff'.

- Clinicians have a responsibility to a) guide patients to appropriate sources of information on the internet; and b) help patients personalise the information gleaned from the internet to their own personal circumstances.

\title{
The nature and quality of periodontal related patient information on the world-wide web
}

\author{
I. G. Chestnutt ${ }^{1}$
}

Background The internet provides a potentially invaluable source of health education material for members of the public.

Objectives This study aimed to investigate the nature and quality of periodontal-related patient information on the world-wide web.

Methods The search term ' "gum disease" AND "patient information" ' was entered into three common internet search engines. The top 50

sites identified by each search engine were downloaded and assessed for authorship, content, accord with accepted dental practice, currency, and references to peer reviewed data.

Results The search term returned in excess of 3,615 hits across the three search engines. Of the top 150 identified, 68 were deemed relevant to patients seeking information on periodontal disease. Of these, 17 were duplicated either within or between search engines, therefore the following analysis is based on the remaining 51 sites. The majority of sites (25) were authored by individual dental practitioners, with sites originating in the USA predominating (80\%). Information on the aetiology, signs and symptoms, and treatment options for periodontal disease was provided by 38, 38, and 29 sites respectively. This information was deemed in accordance with conventional periodontal practice in 27,33 , and 23 cases respectively. Only seven sites indicated the currency of the information and five provided peer-reviewed references for further reading. The rank order identified by the search engines meant that sites providing comprehensive and authoritative information, were not readily differentiable from sites whose primary objective was promoting attendance at specific dental practices.

Conclusions Judicious and active support from professionals is required to aid patients' interpretation of the information identified and to direct them to the most instructive sites.

The potential of the internet to influence how health professionals interact with patients and how healthcare is delivered is enormous and has a number of important considerations for den-

${ }^{1}$ Senior Lecturer and Hon. Consultant in Dental Public Health, Dental Public Health Unit University of Wales College of Medicine.

${ }^{*}$ Correspondence to: Ivor G. Chestnutt, Dental Public Health Unit, University of Wales College of Medicine, Dental School, Health Park, Cardiff CF14 4XY

Email:chestnuttig@cardiff.ac.uk

\section{Refereed paper}

Received 27.02.02; Accepted 17.07.02

$\odot$ British Dental Journal 2002; 192: 657-659 tistry. The world-wide web (WWW) is rapidly becoming an important source of information for patients. Possibly not since Guggenheim's development of the printing press or arguably Baird's invention of the television, has there been such an opportunity to educate and illuminate the masses. ${ }^{1}$

Data from the Office for National Statistics indicate that by April 2001, 51\% of the UK adult population claimed to have accessed the internet. ${ }^{2}$ This equates to some 23 million individuals, of whom just under $80 \%$ had been 'on-line' within the past month. Interest in health information on the WWW is vast - within 4 weeks of operation, the NHS Direct Online site had attracted over 7 million hits. ${ }^{3}$ In late 1998, the National Library of Medicine (NLM) decided to include the public among its primary audiences and initiated a formal programme to encourage consumer access to health information via the NLM website. The establishment of MEDLINEplus, designed for use by public librarians and the general public, provides web users with access to reviewed sources of information from NLM. In the year from April 1999 to April 2000, figures recording use indicate that the number of monthly hits on the MEDLINEplus site rose steadily from just under 200,000 to 1.65 million. ${ }^{4}$

The successful management of periodontal disease requires that patients have a basic understanding of the disease process and over a protracted time period, can maintain an adequate level of plaque control to limit disease progression. Thus, health education and the provision of information to patients is a core component of periodontal practice. However, the nature and quality of information which patients may encounter on a simple search of the WWW has not been established.

This study aimed, using a simple search strategy, to investigate the nature and quality of periodontal-related patient information on the world-wide web.

\section{METHOD}

In July 2001, three commonly accessed search engines/directories, (Excite, Yahoo and Hotbot) were used to search the internet. Search engines are of two basic types but compile their listings from the internet in radically different ways. Crawler based search engines (such as Hotbot), create their listings automatically. In contrast, search directories (such as Yahoo), rely on a manually complied listing. More recently developed engines (eg Google) are hybrids, combining both methods. 
The three used in this study were selected on the basis that they were commonly available, included both a search engine and a search directory and had been used in a recent study by Sacchetti and colleagues ${ }^{5}$ who investigated the availability of internet information on a select urologic topic (Viagra). In the time since this search was conducted (July 2001), other search engines such as Google, Lycos and Alta Vista have become popular. It is likely that they would yield similar results.

The search term " "gum disease" AND "patient information", was entered into each engine and the number of 'hits' recorded. This simple search methodology was adopted without further refinement to simulate the likely outcome of a search conducted by a patient with limited dental, internet or computer knowledge.

The URL (unique resource locator) of the top 50 sites identified by each engine were recorded. The top 50 returns were chosen as it was judged unlikely that the average user would search beyond the first 50 sites returned by a given search. These sites were subsequently downloaded and scored for content and quality. To evaluate websites fully, the entire site was read and scored, not just the particular page identified by the search.

The search results were first reviewed for their relevance to patients seeking information on periodontal disease, and duplicate returns between and within the three search engines were identified. Irrelevant and duplicate sites were removed. Standards based on the quality criteria described by Adelhard ${ }^{6}$ were then used to score the content of the relevant sites. Each website was evaluated to determine: authorship and author's affiliation; an indication that the site had been peer-reviewed; the country of origin of the site; the presence of advertisements (and whether these promoted dental practices or dental products); the currency of the information as indicated by when the site was posted or last updated; the presence of references or further authoritative reading; and warning or disclaimers regarding the information posted. To determine the usability of each site, the number of links to be followed from the URL identified by the search engine, to access the information was noted.

The nature of the clinical information presented was recorded from both a quantitative and qualitative perspective. Clinical information was recorded under three headings, aetiology of periodontal disease, signs and symptoms of periodontal disease and treatment options.

A subjective judgement was made by the author on whether the information presented was in line with conventional periodontal practice. The standard was set by reference to standard periodontal texts ${ }^{7,8}$ and current curricular content in UK undergraduate teaching of periodontology. Finally, advice on the necessity of seeking professional care in the presence of the signs and symptoms of periodontal disease was also recorded.

Data were extracted to a spreadsheet and simple descriptive statistics conducted using SPSS (Chicago, Illinois).

\section{RESULTS}

\section{Non-clinical data}

\section{- Non-relevant and duplicate sites}

The initial searches using the three search engines/directories returned in excess of 3,615 hits; 2,807, 208 and >600 being returned by Excite, Yahoo and Hotbot respectively. Taking the top 50 sites identified by each query, 68 of the 150 (45.3\%) were deemed of relevance to patients seeking information on periodontal disease. Reasons for recoding sites as not of relevance included: link pages to other sites (24), pages focusing primarily on other dental diseases (16) and information majoring on other medical diseases (12). Of the 68 sites providing information on periodontal disease, 17 were duplicated either between or within search engines and the following analysis is therefore based on the remaining 51 sites.

\section{- Authorship of sites}

The majority of sites were attributable to individual general dental practices (25) with a further 12 sites authored by group practices or consortia. Organisations such as the American Dental Association accounted for five sites with academic authors and commercial sources accounting for only two sites each. In five of the 51 cases no author was identifiable.

\section{- Referencing, review and currency of information}

In only one case was it possible to discern that the information presented on the site had been subjected to academic peer review. Of the 51 sites, seven included a disclaimer or note of caution to readers in interpreting the information presented. Further reading or reference to peer reviewed information was provided at five sites. The currency of the information was indicated at the seven sites which included information on the date of posting or updating of the information.

\section{- Country of origin}

The great majority of sites, $41(80.4 \%)$ originated in the USA, the remaining sites being distributed between the UK (2), and Canada, India, Ireland and Sweden which each contributed a single site. In four cases the country of origin of the site was not readily discernible.

\section{- Advertising}

In only four cases was there direct placement of advertisements, of which two were promoting specific dental products. However, 36 of the 51 sites analysed were deemed to be promoting attendance at a specific dental practice or group of practices.

\section{- Ease of access}

Access to the information was in most cases readily available, accessible by following no more than three links in 44 of the 51 cases.

\section{Clinical information}

\section{- Quantitative determination of clinical information}

The aetiology of periodontal disease was discussed by 38 of the 51 sites analysed and an identical number also provided information on signs and symptoms. Disease treatment and management was discussed at 29 (56.9\%) sites. A detailed breakdown of the nature of the information provided is recorded in Table 1.

\section{- Qualitative assessment of clinical information}

The results of the qualitative assessment of clinical information are presented in Table 2 .

Finally, 32 of the 51 sites analysed indicated the need to seek professional care for periodontal disease. In seven cases, the website contained a disclaimer or an indication that the authors did not accept responsibility for the information presented.

\section{DISCUSSION}

The dentist-patient relationship is changing. Today's increasingly health conscious patients have at their disposal, via the internet, a vast repository of information. The WWW has the potential to empower patients and to enable them to make more informed decisions with regard to their oral health and about therapeutic options than ever before. However, the fact that a simple search for information on 'gum disease' returned in excess of 3,615 'hits' gives credence to Eysenbach's ${ }^{9}$ claim that the WWW has the potential to create an environment of chaos and information overload.

Quality criteria have been published to aid evaluation of medical internet sites. ${ }^{6}$ However, these relate more directly to 'academic' published work rather than information directed primarily at laypersons. In common with similar studies on medical top- 


\begin{tabular}{|c|c|c|}
\hline & Discussed $n(\%)$ & Not discussed n (\%) \\
\hline \multicolumn{3}{|c|}{ Aetiology of periodontal disease } \\
\hline \multicolumn{3}{|c|}{ Discussed the role of the following in the aetiology of periodontal disease: } \\
\hline Microbiology & $29(56.9)$ & $22(43.1)$ \\
\hline Plaque / Calculus & $29(56.9)$ & $22(43.1)$ \\
\hline Host immune response & $13(25.5)$ & $38(74.5)$ \\
\hline Tobacco & $10(19.6)$ & $41(80.4)$ \\
\hline Diabetes & 10 (19.6) & $41(80.4)$ \\
\hline \multicolumn{3}{|l|}{ Signs and symptoms } \\
\hline \multicolumn{3}{|c|}{ Discussed the following as signs and symptoms of periodontal disease: } \\
\hline Bleeding & $35(68.6)$ & $16(31.4)$ \\
\hline Swollen gingivae & $34(66.7)$ & $17(33.3)$ \\
\hline Colour & $33(64.7)$ & $18(35.3)$ \\
\hline Recession & $27(52.9)$ & $24(47.1)$ \\
\hline Loose teeth & $34(66.7)$ & $17(33.3)$ \\
\hline Drifting teeth & $24(47.1)$ & $27(52.9)$ \\
\hline Pain & $22(43.1)$ & $29(59.6)$ \\
\hline Halitosis & $29(56.9)$ & $22(43.1)$ \\
\hline \multicolumn{3}{|l|}{ Treatment options } \\
\hline \multicolumn{3}{|c|}{ Discussed the following as options in the management of periodontal disease: } \\
\hline Self-care & $22(43.1)$ & $29(59.6)$ \\
\hline Nonsurgical therapy & $22(43.1)$ & $29(59.6)$ \\
\hline Surgical therapy & $18(35.3)$ & $33(64.7)$ \\
\hline Advanced therapy & $10(19.6)$ & $41(80.4)$ \\
\hline \multicolumn{3}{|c|}{ eg guided tissue regeneration / implants } \\
\hline
\end{tabular}

ics, ${ }^{5,10,11}$ this investigation has demonstrated that the quality of the information returned was variable. Although some potentially very useful sites for patients seeking information about periodontal disease were identified, (eg that hosted by the American Dental Association [www.ada.org/public/faq/gums.html\#signs] and the American Academy of Periodontology [www.perio.org/consumer/faq.htm]), the simple search strategy employed in this study failed to give prominence to these, amongst the weaker sites. In other words, the order in which the sites were identified did not relate to their quality or potential usefulness. The majority of sites returned were hosted either by individual dental practices or consortia of practices in North America. These sites were not dedicated specifically to information on periodontal disease but rather were designed to promote attendance at that practice or to provide information for patients attending the practice.

In the majority of cases, the information presented was in accord with accepted teaching. ${ }^{7,8}$ Perhaps reassuringly, only in a minority of cases was the information contained on the websites factually incorrect (Table 2). However, on some occasions the facts presented verged on 'shock tactics'. For example, in advocating alternative periodontal therapy, one site reported,

'Dentists and periodontists often recommend gum surgery. Surgery may require up to twenty costly office visits. As many as forty injections, thirty incisions and multiple stitches may be necessary. Post surgical pain and a lengthy recovery period are common....'

This site goes on to suggest that by following the recommended course of action ...

'He avoided gum surgery and saved over three thousand dollars on dental bills'12

\begin{tabular}{|c|c|c|c|}
\hline & $\begin{array}{l}\text { Information agrees } \\
\text { with accepted dental } \\
\text { practice }\end{array}$ & $\begin{array}{l}\text { Information partially } \\
\text { agrees with accepted } \\
\text { dental practice }\end{array}$ & $\begin{array}{l}\text { Information at } \\
\text { variance with } \\
\text { accepted dental } \\
\text { practice }\end{array}$ \\
\hline Aetiology $(n=38)$ & 27 & 7 & 4 \\
\hline Signs and symptoms $(n=38)$ & 33 & 5 & 0 \\
\hline Treatment options $(n=29)$ & 23 & 4 & 2 \\
\hline
\end{tabular}

This study has revealed that reasonable, quality information, of value to patients concerned about periodontal disease is available on the WWW. However, locating this information may not be straightforward when using a simple search strategy likely to be employed by the informed but non-professional lay user. Patients need help from their dentist or dental hygienist to sort the 'wheat from the chaff'. There are a number of consumer information gateways increasingly available. These comprise sites which will direct patients to internet resources that at least meet some form of quality criteria. Mention has already been made of the MEDLINEplus site (http://www.nlm.nih.gov/medlineplus). Others have as their objective, a comprehensive listing of patient orientated websites such as (http://www.healthfinder.gov), which is North American in focus or (http://www.patient.co.uk) in the UK. More specific, oral health education related information can be found at sites such as NHS Direct Online, (http://www.nhsdirect.nhs.uk), or the excellent resource maintained by the Health Education Board for Scotland, (http://www.hebs.scot.nhs.uk). Dental practitioners and those involved in oral health education and promotion should be able to direct patients seeking information to suitable sites.

Finally, patients vary greatly in their susceptibility to periodontal disease, ${ }^{13}$ and a major consideration is how patients reading about periodontal disease on the WWW can personalise the information presented, to their individual circumstances. Bleeding and swollen gingivae may be the manifestations of a simple chronic gingivitis or advanced periodontitis. Patients should be aware that information obtained from the internet on periodontal disease is not a substitute for seeing their own dental practitioner. In the present investigation, only 32 of the 51 sites indicated the need to seek further professional advice in the presence of the signs and symptoms of periodontal disease.

\section{CONCLUSIONS}

To summarise, members of the dental team should be aware that although there is a vast amount of health related information on the internet, patients may experience difficulty locating the most useful sites. They should therefore be in a position to direct interested patients to the most relevant material or to selected information gateways.

Most importantly, the increasing availability of information does not detract from the clinicians' primary responsibility of providing personalised information on the aetiology, prevention and management of periodontal disease, tailored to the patient's own personal circumstances.

1. Chestnutt I G. Patient Information on the internet - what are the issues? Community Dent Health 2001; 18: 205-206.

2. Office for National Statistics. National Statistics Omnibus Survey http://www.statistics.gov.uk/statbase 2001 Accessed 27/07/01.

3. Killey R. Finding health information on the internet: Health consumers. Hospital Med 2000; 61: 799-801.

4. National Library of Medicine. MEDLINEplus http://www.n/m.nih.gov/pubs/staffpubs/lo/medlineplus/sld001.htm 2000 Accessed 27/07/01.

5. Sacchetti P, Zvara P, Plante M K. The internet and patient education - resources and their reliability: focus on a select urologic topic. Urology 1999: 53: 1117-1120.

6. Adelhard K, Obst O. Evaluation of medical internet sites. Meth Inform Med 1999; 38: $75-79$.

7. Jenkins W M M, Allan C J. Periodontics: a synopsis. Oxford: Wright, 1999.

8. Manson J D, Eley B M. Periodontal Diseases. 4th ed. Oxford: Wright, 2000.

9. Eysenbach $\mathrm{G}$. Rating information on the internet can empower users to make informed decisions. Br Med J 1999; 319: 385-386.

10. Soot L C, Moneta G L, Edwards J M. Vascular surgery and the internet: A poor source of patient-orientated information. J Vasc Surg 1999; 30: 84-91.

11. Harmon D, Duggan M, Flynn N. Anaesthesia on the world-wide web: is reliable patient information available in the internet? Anaesthesia 2000; 55: 728-729.

12. Hart J. How you can avoid gum surgery and keep your teeth and save on dental bills. 2001. www.gum-disease-teeth-oral-ada.com. Accessed 11/07/01.

13. Papapanou P N. Periodontal diseases: epidemiology. Ann Periodontol 1996; 1:136. 